\title{
Synthesis, Characterization and Antibacterial Activity of Mixed Ligand (HL) Complexes Mn(ll), Co(ll), Ni(ll), Zn(ll), Cd(ll) and $\mathrm{Hg}(11)$ with Azide $\left(\mathrm{N}_{3}^{-}\right)$
}

\author{
Jassim S. Sultan, Sajed M. Lateaf, Dhuha K. Rashid \\ Department of Chemistry, College of Education for pure Sciences, Ibn Al-Hiatham, University of Baghdad, \\ Baghdad, Iraq \\ Email: ja.sultan@yahoo.com
}

Received 12 June 2015; accepted 5 October 2015; published 8 October 2015

Copyright (C) 2015 by authors and Scientific Research Publishing Inc.

This work is licensed under the Creative Commons Attribution International License (CC BY).

http://creativecommons.org/licenses/by/4.0/

cc) (i) Open Access

\begin{abstract}
The complexes of mixed ligand (HL) as primary ligand with azide ion ( $\left.\mathrm{N}_{3}^{-}\right)$as co-ligand with $\mathrm{Mn}(\mathrm{ll})$, $\mathrm{Co}(\mathrm{II}), \mathrm{Ni}(\mathrm{ll}), \mathrm{Zn}(\mathrm{ll}), \mathrm{Cd}(\mathrm{ll})$ and $\mathrm{Hg}(\mathrm{ll})$ were prepared via reaction metal (II) chloride salt with ligand (HL) and sodium azide $\left(\mathrm{NaN}_{3}\right)$ using 1:2:2 mole ratio in ethanol solvent, respectively. The complexes of mixed ligand (HL) were characterized by elemental microanalysis (C.H.N), atomic absorption chloride content, molar conductance, magnetic susceptibility, melting point, FTIR and UV-Vis spectral data. The anti bacterial activity with four kinds of bacteria, Staphylococcus aureus, Bacillus, Escherichia coli and Pseudomonas aureus was studied.
\end{abstract}

\section{Keywords}

\section{4-Aminoantipyrine, Glyoxylic Acid, Sodium Azide, Schiff Base, Azido Mixed Ligand}

\section{Introduction}

The chemistry of Schiff bases metal complexes is of interest because these species display a variety of reactivity mode and they possess catalytic and biological activity [1]. Metal complexes of nitrogen-oxygen chelating agents derived from 4-aminoantipyrine Schiff bases have been studied extensively due to their pronounced applications in biological, clinical, analytical and pharmacological areas [2]-[4].

4-Aminoantipyrine, an antipyretic agent [5] is one of the pyrazole derivatives. Numerous synthetic compounds containing pyrazole moiety have been focused in the field of medicinal chemistry [6] because of their 
pharmacological, photographic, catalytic and liquid crystals applications [7] [8]. Glyoxylic acid and its derivatives play important roles in natural processes participating in the glyoxylate cycle which functions in plants and some microorganisms [9]. It has been widely used in organic synthesis for the manufacture of intermediate products in pharmaceutical [10]. The spectrophotometric methods are usually based on the reactivity of the aldehyde group [11] [12]. Azido-mixed ligand complexes particular interest has been focused on the azido ligand $\left(\mathrm{N}_{3}^{-}\right)$not only for its efficiency in ferromagnetic or antiferromagnatic coupling but also for its diversity in coordination modes, $\mu-1,1$, (end-on EO), $\mu-1,3$ (end-end, EE), $\mu-1,1,1, \mu-1,1,3$ or still other modes and polymetric bridging network, and a large number of azido-bridged complexes with different dimensionalities and various topologies have been reported in the literature [13] [14]. Polynuclear complexes and coordination polymer in which paramagnetic metal ions are bridged by short ligands have attracted great attention in recent years. The studies focusing on better understanding fundamental magnetic applications, a large number of a zidebridged transition metal complexes, have been reported [15].

\section{Experimental}

All chemicals were purchased from BDH, and used without further purifications.

\subsection{Instrumentation}

FTIR spectra were recorded in $\mathrm{KBr}$ on Shimadzu-8300 Spectrophotometer in the range of (4000 - $400 \mathrm{~cm}^{-1}$ ). The electronic spectra in $\mathrm{H}_{2} \mathrm{O}$ were recorded using the UV-Visible spectrophotometer type (spectra 190 - 900 $\mathrm{nm})$ CECIL, England, with quartz cell of $(1 \mathrm{~cm})$ path length. The melting point was recorded on Gallen kamp Melting point Apparatus.

The Conductance Measurements were recorded on W. T. W. conductivity Meter. The metal contents of the complexes were determined by atomic absorption (A. A.) technique using a Shimadzu PR-5 with Orphic Printer atomic absorption spectrophotometer. Balance Magnetic Susceptibility model MSB-MLI was conducted for measuring the magnetic susceptibility.

The characterization of the new ligand $(\mathrm{L})$ is achieved by $1 \mathrm{H}$ and ${ }^{13} \mathrm{C}-\mathrm{NMR}$ spectra were recorded by using a Bruker 300 MHZ (Switzerland). Chemical Shift of all ${ }^{1} \mathrm{H}$ and ${ }^{13} \mathrm{C}$-NMR spectra were recorded in $\boldsymbol{\delta}(\mathrm{ppm})$ unit downfield from internal reference tetramethylsilane (TMS), using $\mathrm{D}_{2} \mathrm{O}$ as a solvent. Elemental analysis for carbon, hydrogen was performed using a Euro Vector EA 3000 A Elemental Analysis (Italy).

\subsection{Synthesis of Ligand and Complexes}

\subsubsection{Synthesis of Ligand (1,5-Diemthyl-3-oxo-2-phenyl-2,3-dihydro-1H-pyrazol-4-ylimino) (HL)}

A solution of (4-AAP) (0.203 g, 1 mmole) in ethanol ( $20 \mathrm{ml})$, and a few drop of $48 \%$ of $\mathrm{HBr}$, was added to a solution of (Glyoxylic acid) $(0.074 \mathrm{~g}, 1 \mathrm{mmol})$ in ethanol $(10 \mathrm{ml})$. The mixture was refluxed for $(6 \mathrm{hr})$ with stirring. The resulting was an orange solution allowed to cool and dried at room temperature, then washed with ethanol and re-crystallization to the precipitate with methanol/ $\mathrm{H}_{2} \mathrm{O}$ to give orange crystals during $(24 \mathrm{hr})$, m.p. $(128-132)^{\circ} \mathrm{C}$.

\subsubsection{Synthesis of $\left[\mathrm{Co}(\mathrm{HL})_{2}\left(\mathrm{~N}_{3}\right)_{2}\right] \cdot 2 \mathrm{H}_{2} \mathrm{O}$ Complex}

A solution of (HL) (0.518 g, 2 mmole) in ethanol (5 ml) and a solution of $\mathrm{NaN}_{3}(0.13 \mathrm{~g}, 2 \mathrm{mmol})$ in ethanol (5 $\mathrm{ml})$ were added to a stirred solution of $\mathrm{CoCl}_{2} \cdot 6 \mathrm{H}_{2} \mathrm{O}(0.238 \mathrm{~g}, 1 \mathrm{mmol})$ in ethanol $(5 \mathrm{ml})$. The resulting mixture was stirred for $(1 \mathrm{hr})$. Then the mixture was filtered and dried then the precipitate was washed with an excess of ethanol and dried at room temperature during $(24 \mathrm{hr})$. A pale green crystals were obtained, m.p. $(116-121)^{\circ} \mathrm{C}$. A similar method was used to prepare of $\mathrm{Mn}(\mathrm{II}), \mathrm{Ni}(\mathrm{II}), \mathrm{Zn}(\mathrm{II}), \mathrm{Cd}(\mathrm{II})$ and $\mathrm{Hg}(\mathrm{II})$ mixed ligand complexes.

\section{Results and Discussion}

\subsection{Characterization of Ligand (HL)}

In this study, new Schiff base ligand (HL) type (NOO) donor atoms was synthesized according to the used method shown in Scheme 1.

Spectroscopic methods [FT-IR, UV-Vis, ${ }^{1} \mathrm{H}$ NMR, ${ }^{13} \mathrm{C}$ NMR] along with melting point and elemental microanalysis C.H.N. were used to characterization the new ligand (HL). 


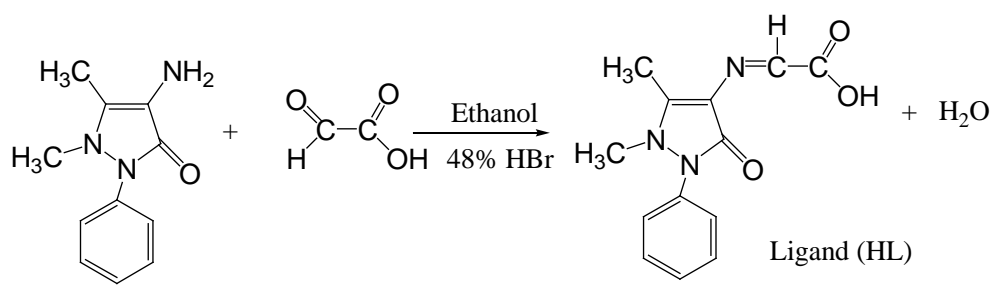

Scheme 1. Synthesis route of ligand (HL).

\subsection{1. ${ }^{1} \mathrm{H}$ NMR Spectral Data}

The ${ }^{1} \mathrm{H}-\mathrm{NMR}$ spectrum of ligand (HL) shows single peaks attributed to two methyl groups appeared at range $(\delta 1.06-\delta 2.91) \mathrm{ppm}$. The strong signal obtained at $(\delta 3.35) \mathrm{ppm}$. due to DMSO-d $\mathrm{d}_{6}$. The weak peak at ( $\left.\delta 8.14\right)$ ppm was attributed to proton of azomethine group $(-\mathrm{N}=\mathrm{CH}-)$. Single weak peak attributed to proton of $(-\mathrm{COOH})$ appeared at (89.30) ppm. The multiple chemical shifts around (6.98 - 7.47) ppm may assigned to aromatic protons. The two weak signals at $\delta(3.04)$ and $\delta(3.14)$ ppm refer to water molecule of DMSO [16].

\subsection{2. ${ }^{13} \mathrm{C}$ NMR Spectral Data}

${ }^{13} \mathrm{C}$ NMR spectrum of ligand (HL) shows chemical shifts at (15.54) ppm and (18.35) ppm refer to $\mathrm{C}_{5}$ and $\mathrm{C}_{6}$ for two $\mathrm{CH}_{3}$ group respectively. The chemical shifts at (61.79) ppm and (88.32) ppm were attributed to $\mathrm{C}_{3}$ and $\mathrm{C}_{4}$ of $\mathrm{C}=\mathrm{C}$ in 4-AAP ring respectively. Signals related to aromatic carbon $\left(\mathrm{C}_{7}-\mathrm{C}_{12}\right)$ were detected at range (126.04 130.89) ppm. The chemical shift of $C_{1}$ for carboxylic group appeared as expected downfield at (170.25) ppm. The two chemical shift at (165.51) ppm and (135.47) ppm were attributed to $\mathrm{C}_{2}$ for azomethine group (-N=CH-) and $\mathrm{C}_{13}$ to $\mathrm{C}=\mathrm{O}$ for 4-AAP respectively. Finally, the chemical shift at (40.86) ppm is due to DMSO $\mathrm{d}_{6}$ [17].

\subsection{Characterization of Mixed Ligand (HL) Complexes with Azide $\left(\mathbf{N}_{3}^{-}\right)$}

The complexes of mixed ligand, (HL) as primary ligand with azide ion $\left(\mathrm{N}_{3}^{-}\right)$as co-ligand with $\mathrm{Mn}(\mathrm{II}), \mathrm{Co}(\mathrm{II})$, $\mathrm{Ni}(\mathrm{II}), \mathrm{Zn}(\mathrm{II}), \mathrm{Cd}(\mathrm{II})$ and $\mathrm{Hg}(\mathrm{II})$ were prepared via reaction metal (II) chloride salt with ligand (HL) and sodium azide $\left(\mathrm{NaN}_{3}\right)$ using 1:2:2 mole ratio in ethanol solvent respectively. The method of this synthesis shows in Scheme 2 and Scheme 3.

Spectroscopic methods [FT-IR, UV-Vis, A. A.] along with molar conductivity, elemental microanalysis C.H.N, Chloride content, magnetic susceptibility, melting point were used to characterize the prepared mixed ligand complexes. All mixed ligand complexes are stable in solution and soluble in methanol, ethanol, acetone, DMSO and DMF solvents. Some physical properties were listed in Table 1. Elemental microanalysis C.H.N, metal and chloride analysis are in a good agreement with calculated values, Table 2.

\subsubsection{Molar Conductance}

The molar conductance values, Table 1, of the soluble mixed ligand complexes in DMSO solvent in $10^{-3} \mathrm{M}$ solution at room temperature refer to non-electrolytic nature [18].

\subsubsection{Magnetic Susceptibility}

The magnetic susceptibility for all complexes were measured at room temperature and the effective magnetic moment $\left(\mu_{\text {eff }}\right)$ values [19] were listed in Table 3.

\subsubsection{FT-IR Spectral Data}

The IR spectra of mixed ligand complexes Co(II), Ni(II), Zn(II), Cd(II), and Hg(II), exhibited band at (1616) $\mathrm{cm}^{-1}$, (1624) $\mathrm{cm}^{-1}$, (1614) $\mathrm{cm}^{-1}$, (1616) $\mathrm{cm}^{-1}$, and (1635) $\mathrm{cm}^{-1}$, respectively, refers to stretching frequency $v \mathrm{C}=\mathrm{O}$ of 4-AAP ring, which was shifted to lower frequency when its comparison with that of the free ligand (HL), showing that the coordination between oxygen atom of this group with metal ion has happened [20]. The IR spectrum of mixed ligand complex of Mn(II), showed no change in position of the stretching frequency of $v \mathrm{C}=\mathrm{O}$ ring when it compare of free ligand, indicating that the oxygen atom of $(\mathrm{C}=\mathrm{O})$ ring wasn't involved in coordination with $\mathrm{Mn}(\mathrm{II})$. The detected bands at (1593) $\mathrm{cm}^{-1}$, (1591) $\mathrm{cm}^{-1}$, (1593) $\mathrm{cm}^{-1}$, (1591) $\mathrm{cm}^{-1}$, (1591) $\mathrm{cm}^{-1}$, and (1591) $\mathrm{cm}^{-1}$ in the IR spectra of all mixed ligand complexes refer to stretching frequency of imine 
<smiles>Cc1c(/N=C/C(=O)[OH2+])c(=O)n(-c2ccccc2)n1C</smiles>

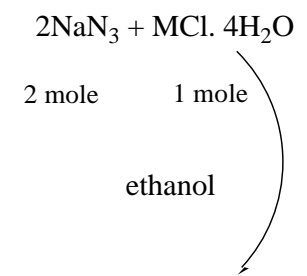

$\Gamma$

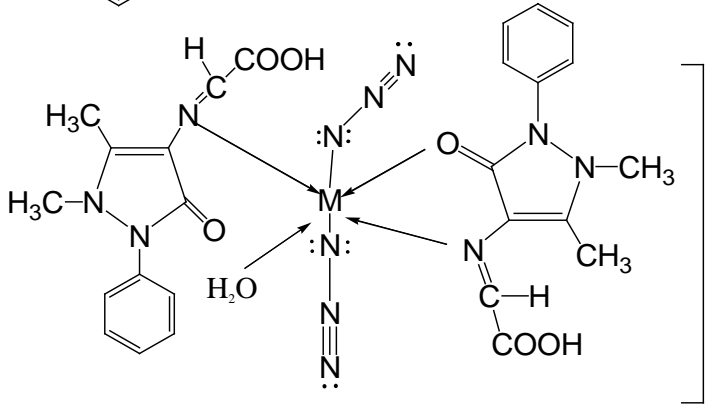

reflux

Where:M $=\mathrm{Co}, \mathrm{X}=6, \mathrm{~m}=2$

$\mathrm{Ni}, \mathrm{X}=6, \mathrm{~m}=3$

$\mathrm{Zn}, \mathrm{X}=0, \mathrm{~m}=3$

$\mathrm{Cd}, \mathrm{X}=1, \mathrm{~m}=2$

$\mathrm{mH}_{2} \mathrm{O}+2 \mathrm{NaCl} \quad \mathrm{Hg}, \mathrm{X}=0, \mathrm{~m}=3$

Scheme 2. Synthesis route of mixed ligand complexes for $\mathrm{Co}(\mathrm{II}), \mathrm{Ni}(\mathrm{II}), \mathrm{Zn}(\mathrm{II}), \mathrm{Cd}(\mathrm{II})$ and $\mathrm{Hg}(\mathrm{II})$.
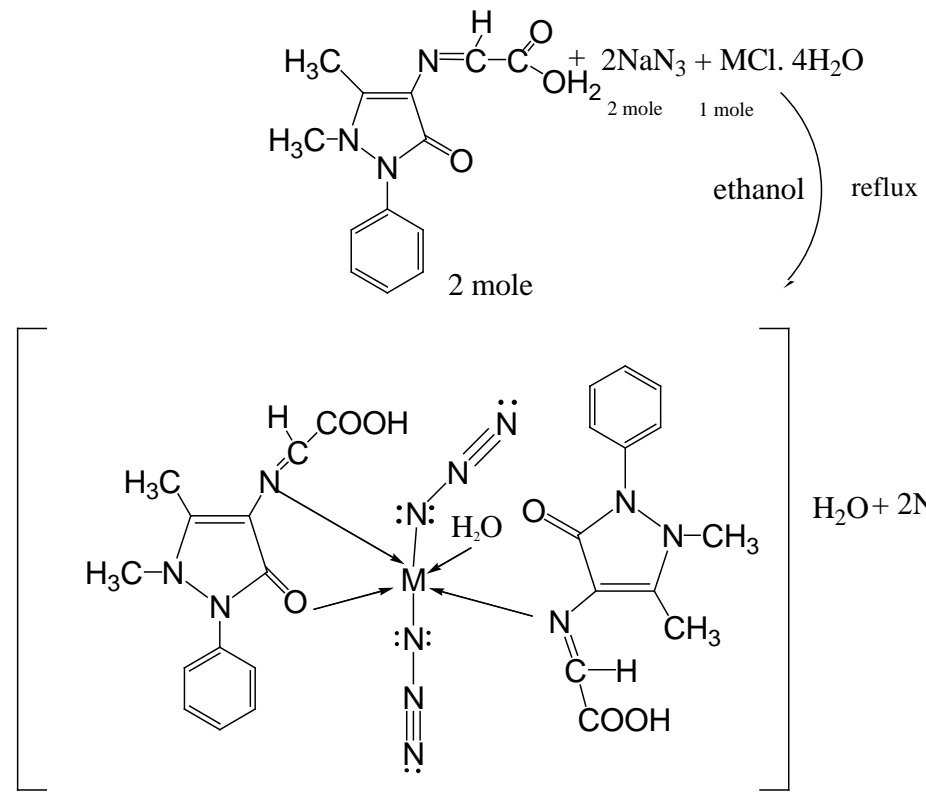

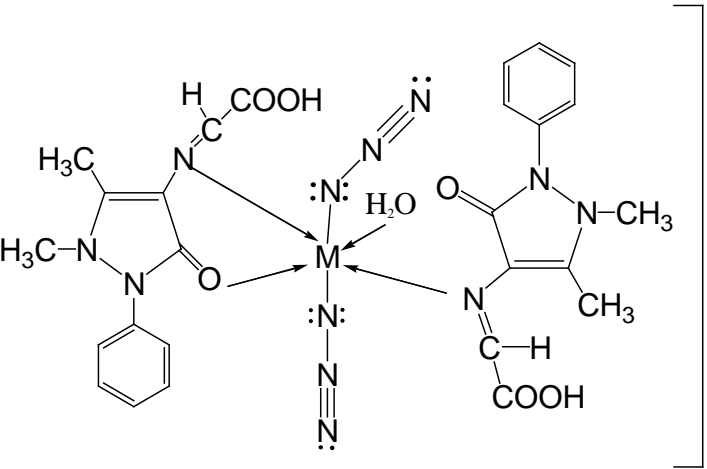

$\mathrm{H}_{2} \mathrm{O}+2 \mathrm{NaCl}$

Scheme 3. Synthesis route of mixed ligand (HL) complex for $\left[\mathrm{Mn}(\mathrm{HL})_{2}\left(\mathrm{~N}_{3}\right)_{2}\left(\mathrm{H}_{2} \mathrm{O}\right)_{2}\right] \cdot \mathrm{H}_{2} \mathrm{O}$.

Table 1. Some physical properties and molar conductance (M.C) of mixed ligand (HL) complexes.

\begin{tabular}{|c|c|c|c|c|c|}
\hline Complexes & M.wt g/mol & Yield \% & Colour & M. P. ${ }^{\circ} \mathrm{C}$ & M.C $\mathrm{ohm}^{-1} \cdot \mathrm{cm}^{2} \cdot \mathrm{mol}^{-1}$ \\
\hline$\left[\mathrm{Mn}(\mathrm{HL})_{2}\left(\mathrm{~N}_{3}\right)_{2}\left(\mathrm{H}_{2} \mathrm{O}\right)\right] \cdot \mathrm{H}_{2} \mathrm{O}$ & 711 & 78 & Dark green & 100 & 12.22 \\
\hline$\left[\mathrm{Co}(\mathrm{HL})_{2}\left(\mathrm{~N}_{3}\right)_{2}\right] \cdot 2 \mathrm{H}_{2} \mathrm{O}$ & 697 & 91 & Pale green & 120 & 9.42 \\
\hline$\left[\mathrm{Ni}(\mathrm{HL})_{2}\left(\mathrm{~N}_{3}\right)_{2}\right] \cdot 3 \mathrm{H}_{2} \mathrm{O}$ & 714.7 & 92 & Pale olive & 122 & 15.58 \\
\hline$\left[\mathrm{Zn}(\mathrm{HL})_{2}\left(\mathrm{~N}_{3}\right)_{2}\right] \cdot 3 \mathrm{H}_{2} \mathrm{O}$ & 721.4 & 88 & Pale orange & dec. 108 - 112 & 7.97 \\
\hline$\left[\mathrm{Cd}(\mathrm{HL})_{2}\left(\mathrm{~N}_{3}\right)_{2}\right] \cdot 2 \mathrm{H}_{2} \mathrm{O}$ & 750.4 & 89 & Pale orange & 103 & 9.21 \\
\hline$\left[\mathrm{Hg}(\mathrm{HL})_{2}\left(\mathrm{~N}_{3}\right)_{2}\right] \cdot 3 \mathrm{H}_{2} \mathrm{O}$ & 856.6 & 92 & Pale orange & 102 & 9.16 \\
\hline
\end{tabular}


Table 2. Elemental microanalysis of mixed ligand (HL) complexes.

\begin{tabular}{cccccc}
\hline \multirow{2}{*}{ Complexes } & \multicolumn{5}{c}{ Found, (cal cu.)\% } \\
\cline { 2 - 6 } & $\mathbf{C}$ & $\mathbf{H}$ & $\mathbf{N}$ & M & Cl \\
\hline$\left[\mathrm{Mn}(\mathrm{HL})_{2}\left(\mathrm{~N}_{3}\right)_{2}\left(\mathrm{H}_{2} \mathrm{O}\right)\right] \cdot \mathrm{H}_{2} \mathrm{O}$ & $(43.88) 43.06$ & $(4.50) 3.85$ & $(23.62) 23.84$ & $(7.73) 7.26$ & Nil \\
{$\left[\mathrm{Co}(\mathrm{HL})_{2}\left(\mathrm{~N}_{3}\right)_{2}\left(\mathrm{H}_{2} \mathrm{O}\right)_{2}\right] \cdot \mathrm{H}_{2} \mathrm{O}$} & $(44.76) 44.03$ & $(4.01) 4.26$ & $(24.10) 23.67$ & $(8.46) 8.01$ & Nil \\
{$\left[\mathrm{Ni}(\mathrm{HL})_{2}\left(\mathrm{~N}_{3}\right)_{2}\right] \cdot 3 \mathrm{H}_{2} \mathrm{O}$} & $(43.65) 42.86$ & $(4.47) 3.82$ & $(23.50) 22.64$ & $(8.21) 7.75$ & Nil \\
{$\left[\mathrm{Zn}(\mathrm{HL})_{2}\left(\mathrm{~N}_{3}\right)_{2}\right] \cdot 3 \mathrm{H}_{2} \mathrm{O}$} & $(43.24) 42.75$ & $(4.43) 4.68$ & $(23.28) 22.85$ & $(9.06) 8.42$ & Nil \\
{$\left[\mathrm{Cd}(\mathrm{HL})_{2}\left(\mathrm{~N}_{3}\right)_{2}\right] \cdot 2 \mathrm{H}_{2} \mathrm{O}$} & $(41.57) 40.82$ & $(3.99) 3.52$ & $(22.38) 23.04$ & $(14.97) 14.62$ & Nil \\
{$\left[\mathrm{Hg}(\mathrm{HL})_{2}\left(\mathrm{~N}_{3}\right)_{2}\right] \cdot 3 \mathrm{H}_{2} \mathrm{O}$} & $(36.42) 35.86$ & $(3.73) 3.25$ & $(19.61) 19.18$ & $(23.41) 22.94$ & Nil \\
\hline
\end{tabular}

Table 3. Magnetic susceptibility and $\mu_{\text {eff }}$ (B.M) of some mixed ligand (HL) complexes.

\begin{tabular}{cccccc}
\hline Complexes* & $\mathbf{X}_{\mathbf{g}} \times \mathbf{1 0}^{-\mathbf{6}}$ & $\mathbf{X}_{\mathbf{M}} \times \mathbf{1 0}^{-\mathbf{6}}$ & $\mathbf{X}_{\mathbf{A}} \times \mathbf{1 0}^{-\mathbf{6}}$ & $\boldsymbol{\mu}_{\text {eff. }}(\mathbf{B . M})$ & Suggested structure \\
\hline$\left[\mathrm{Mn}(\mathrm{HL})_{2}\left(\mathrm{~N}_{3}\right)_{2}\left(\mathrm{H}_{2} \mathrm{O}\right)_{2}\right] \cdot \mathrm{H}_{2} \mathrm{O}$ & 18.57 & 13204.65 & 12971.03 & 5.56 & Octahedral \\
{$\left[\mathrm{Co}(\mathrm{HL})_{2}\left(\mathrm{~N}_{3}\right)_{2}\right] \cdot 2 \mathrm{H}_{2} \mathrm{O}$} & 14.08 & 9815.38 & 10049.0 & 4.88 & Octahedral \\
{$\left[\mathrm{Ni}(\mathrm{HL})_{2}\left(\mathrm{~N}_{3}\right)_{2}\right] \cdot 3 \mathrm{H}_{2} \mathrm{O}$} & 4.50 & 3217.88 & 3451.50 & 2.86 & Octahedral \\
\hline
\end{tabular}

group $(-\mathrm{N}=\mathrm{CH}-)$ which were shifted to lower frequency when it compare with that of free ligand showing that the coordination with the metal ions was occurred via nitrogen atom of imine group (-N=CH-) [21]. The IR spectra of all mixed ligand complexes exhibited new strong splited band or new strong singlate band which wasn't observed in the IR spectrum of free ligand (HL), this band was located at $\left(2123 \mathrm{~cm}^{-1}, 2058 \mathrm{~cm}^{-1}\right) \mathrm{Mn}(\mathrm{II})$, $\left(2123 \mathrm{~cm}^{-1}, 2102 \mathrm{~cm}^{-1}\right) \mathrm{Co}(\mathrm{II}),\left(2121 \mathrm{~cm}^{-1}, 2036 \mathrm{~cm}^{-1}\right) \mathrm{Ni}(\mathrm{II}),\left(2125 \mathrm{~cm}^{-1}, 2036 \mathrm{~cm}^{-1}\right) \mathrm{Hg}(\mathrm{II}),(2065) \mathrm{cm}^{-1}$ for $\mathrm{Zn}(\mathrm{II})$ and $\mathrm{Cd}(\mathrm{II})$, which was attributed to stretching frequency $v_{\text {asy. }}\left(\mathrm{N}_{3}^{-}\right)$. While the new band at $(1311) \mathrm{cm}^{-1}$ for $\mathrm{Mn}(\mathrm{II}) \mathrm{Co}(\mathrm{II})$ and at (1317) $\mathrm{cm}^{-1}$ for $\mathrm{Zn}(\mathrm{II}), \mathrm{Cd}(\mathrm{II})$ and at (1313) $\mathrm{cm}^{-1}$ for $\mathrm{Hg}(\mathrm{II})$, was attributed to stretching frequency $v_{\text {sy. }}\left(\mathrm{N}_{3}^{-}\right)$[22]. These values of $v_{\text {asy. }}\left(\mathrm{N}_{3}^{-}\right)$and $v_{\text {sy. }}\left(\mathrm{N}_{3}^{-}\right)$suggest the coordination between azide ion $\left(\mathrm{N}_{3}^{-}\right)$and metal ion. Further, the two bands observed at (1419) $\mathrm{cm}^{-1}$ and (1369) $\mathrm{cm}^{-1}$ in the IR spectrum of free ligand which are assigned to $v_{\text {asy. }}\left(\mathrm{COO}^{-}\right)$and $v_{\text {sy }}$. $\left(\mathrm{COO}^{-}\right)$respectively, parically no or slightly change on these frequencies at complexation was observed, indicating noninvolvement of this group in coordination with metal ion [23]. In the IR spectra of mixed ligand complexes of Mn(II), Co(II), Ni(II), Zn(II), Cd(II), and $\mathrm{Hg}(\mathrm{II})$ abroad band was observed around (3464) $\mathrm{cm}^{-1}$ to (3390) $\mathrm{cm}^{-1}$ which was assigned to hydrate water molecules [24], while the presence of coordinated water (aqua) in the structure of $\mathrm{Mn}(\mathrm{II})$ complex was suggested by a broad band at (3390) $\mathrm{cm}^{-1}$ and weak band at (931) $\mathrm{cm}^{-1}$ in the IR spectrum of Mn(II) complex, which refer to $v \mathrm{O}-\mathrm{H}$ and $\delta \mathrm{O}-\mathrm{H}$ for $\mathrm{H}_{2} \mathrm{O}$ coordination molecules [25]. The stretching frequency of $v \mathrm{C}=\mathrm{O}$ for carboxylate group in IR spectra for all mixed complexes appeared as overlap with band of $v \mathrm{C}=\mathrm{O}$ ring or as a weak band around (1735) $\mathrm{cm}^{-1}$. Two bands in the region (545 - 505) $\mathrm{cm}^{-1}$ and $(505-445) \mathrm{cm}^{-1}$ typical for the presence of M-N and $\mathrm{M}-\mathrm{O}$ in the IR spectra for all mixed ligand complexes as new bands [26]. These Two bands were not present in the spectrum of free ligand (HL). Other bands in the IR for all complexes were listed in Table 4. These observation in the IR spectra of free ligand and its mixed complexes indicate that the ligand (HL) coordinate with Co(II), $\mathrm{Ni}(\mathrm{II}), \mathrm{Zn}(\mathrm{II}), \mathrm{Cd}(\mathrm{II})$, and $\mathrm{Hg}(\mathrm{II})$ via oxygen atom of $(\mathrm{C}=\mathrm{O})$ ring and nitrogen atom of imine group $(-\mathrm{N}=\mathrm{CH}-)$, behaving bidentate ligand. But its behaves as monodentate ligand when it coordinate with $\mathrm{Mn}$ (II) via nitrogen atom of imine group $(-\mathrm{N}=\mathrm{CH}-)$ only. While the azide ion $\left(\mathrm{N}_{3}^{-}\right)$coordinates with metal ion in all mixed ligand complexes as terminal ligand. According to these fact, the primary oxidation states of the metal ions $\mathrm{Mn}$ (II), $\mathrm{Co}(\mathrm{II}), \mathrm{Ni}(\mathrm{II}), \mathrm{Zn}(\mathrm{II}), \mathrm{Cd}(\mathrm{II})$, and $\mathrm{Hg}(\mathrm{II})$ ware satisfied by the presence of azide ion $\left(\mathrm{N}_{3}^{-}\right)$present inside the coordination sphere, that was proved by molar conductance values which were a good evidence for non-electrolytic complexes.

\subsubsection{UV-Vis Spectral Data}

The electronic spectral data for all mixed ligand complexes are summarized in Table 5, together with electronic 
Table 4. The FT-IR spectral data $\left(\mathrm{cm}^{-1}\right)$ of ligand (HL) and its mixed complexes.

\begin{tabular}{|c|c|c|c|c|c|c|c|c|c|c|c|c|c|c|}
\hline Compounds & $v O-H$ & $\begin{array}{c}v \mathrm{C}=\mathrm{O} \\
\text { ring }\end{array}$ & $\begin{array}{c}v \mathrm{C}=\mathrm{O} \\
\text { carbox. }\end{array}$ & $\begin{array}{l}v N=C \\
\text { imine }\end{array}$ & $\begin{array}{c}v \mathrm{COO}^{-} \\
\text {asy. }\end{array}$ & $\begin{array}{c}v^{\mathrm{COO}^{-}} \\
\text {sy. }\end{array}$ & $\begin{array}{l}v \mathrm{~N}_{3}^{-} \\
\text {asy. }\end{array}$ & $\begin{array}{c}v \mathrm{~N}_{3}^{-} \\
\text {sy. }\end{array}$ & $\begin{array}{l}v \mathrm{C}=\mathrm{C} \\
\text { arom. }\end{array}$ & $\begin{array}{l}v \mathrm{C}-\mathrm{H} \\
\text { arom. }\end{array}$ & $\begin{array}{l}v C-C \\
\text { aliph. }\end{array}$ & $\begin{array}{l}v \mathrm{C}-\mathrm{H} \\
\text { aliph. }\end{array}$ & $v \mathbf{M}-\mathbf{N}$ & vM-O \\
\hline (HL) & 3421 & 1645 & 1724 & 1608 & 1419 & 1369 & - & - & 1586 & 2939 & 1197 & 2900 & - & - \\
\hline$\left[\mathrm{Mn}(\mathrm{HL})_{2}\left(\mathrm{~N}_{3}\right)_{2}\left(\mathrm{H}_{2} \mathrm{O}\right)_{2}\right] \cdot \mathrm{H}_{2} \mathrm{O}$ & 3390 & 1647 & Overlap & 1593 & 1417 & 1369 & $\begin{array}{l}2123 \\
2058\end{array}$ & 1311 & 1570 & 3064 & 1190 & 2927 & 543 & 505 \\
\hline$\left[\mathrm{Co}(\mathrm{HL})_{2}\left(\mathrm{~N}_{3}\right)_{2}\right] \cdot \mathrm{H}_{2} \mathrm{O}$ & 3442 & 1616 & Overlap & 1591 & 1421 & 1371 & $\begin{array}{l}2123 \\
2102\end{array}$ & 1311 & 1573 & Overlap & 1197 & 2935 & 505 & 445 \\
\hline$\left[\mathrm{Ni}(\mathrm{HL})_{2}\left(\mathrm{~N}_{3}\right)_{2}\right] \cdot 3 \mathrm{H}_{2} \mathrm{O}$ & 3390 & 1624 & 1735 & 1593 & 1417 & 1363 & $\begin{array}{l}2121 \\
2036\end{array}$ & 1311 & 1575 & Overlap & 1168 & Overlap & 545 & 475 \\
\hline$\left[\mathrm{Zn}(\mathrm{HL})_{2}\left(\mathrm{~N}_{3}\right)_{2}\right] \cdot 3 \mathrm{H}_{2} \mathrm{O}$ & 3464 & 1614 & 1735 & 1591 & 1417 & 1373 & 2065 & 1317 & 1560 & 3066 & 1141 & 2933 & 542 & 445 \\
\hline$\left[\mathrm{Cd}(\mathrm{HL})_{2}\left(\mathrm{~N}_{3}\right)_{2}\right] \cdot 2 \mathrm{H}_{2} \mathrm{O}$ & 3446 & 1616 & Overlap & 1591 & 1419 & 1375 & 2065 & 1317 & 1558 & 3064 & 1188 & 2930 & 505 & 460 \\
\hline$\left[\mathrm{Hg}(\mathrm{HL})_{2}\left(\mathrm{~N}_{3}\right)_{2}\right] \cdot 3 \mathrm{H}_{2} \mathrm{O}$ & 3346 & 1635 & Overlap & 1591 & 1417 & 1369 & $\begin{array}{l}2125 \\
2036\end{array}$ & 1313 & 1558 & 3064 & 1188 & 2900 & 505 & 453 \\
\hline
\end{tabular}

Table 5. Electronic spectral data of ligand (HL) and its mixed complexes.

\begin{tabular}{|c|c|c|c|c|c|}
\hline Compound & $\lambda \mathbf{n m}$ & $v^{-}\left(\mathrm{cm}^{-1}\right)$ & $\left(\varepsilon_{\max } \operatorname{molar}^{-1} \cdot \mathbf{L} \cdot \mathbf{c m}^{-1}\right)$ & Electronic transition & Suggested structure \\
\hline \multirow{3}{*}{ (HL) } & 276 & 36,231 & 1885 & $\pi \rightarrow \pi^{*}$ & \multirow{3}{*}{-} \\
\hline & 346 & 28,901 & 614 & $\mathrm{n} \rightarrow \pi^{*}$ & \\
\hline & 362 & 27,624 & 500 & $\mathrm{n} \rightarrow \pi^{*}$ & \\
\hline \multirow{6}{*}[\mathrm{Mn}(\mathrm{HL})_{2}(\mathrm{N}_{3})_{2}(\mathrm{H}_{2}\mathrm{O})_{2}]{$\cdot \mathrm{H}_{2} \mathrm{O}$} & 297 & 33,670 & 2370 & Intra-ligand & \multirow{6}{*}{$\mathrm{Oh}$} \\
\hline & 345 & 28,986 & 2042 & Intra-ligand & \\
\hline & 359 & 27,855 & 1324 & Intra-ligand & \\
\hline & 475 & 21,053 & 208 & ${ }^{6} \mathrm{~A}_{1} \mathrm{~g} \rightarrow{ }^{4} \mathrm{~T}_{2} \mathrm{~g}(\mathrm{G})$ & \\
\hline & 638 & 15,674 & 41 & ${ }^{6} \mathrm{~A}_{1} \mathrm{~g} \rightarrow{ }^{4} \mathrm{~T}_{1} \mathrm{~g}(\mathrm{G})$ & \\
\hline & 794 & 12,594 & 7 & ${ }^{6} \mathrm{~A}_{1} \mathrm{~g} \rightarrow{ }^{4} \mathrm{Eg}(\mathrm{D})$ & \\
\hline \multirow{5}{*}[\mathrm{Co}(\mathrm{HL})_{2}(\mathrm{N}_{3})_{2}]{$\cdot 2 \mathrm{H}_{2} \mathrm{O}$} & 286 & 34,965 & 2304 & Intra-ligand & \multirow{5}{*}{ oh } \\
\hline & 345 & 28,986 & 2034 & Intra-ligand & \\
\hline & 362 & 27,624 & 1228 & Intra-ligand & \\
\hline & 650 & 15,387 & 13 & ${ }^{4} \mathrm{~T}_{1} \mathrm{~g} \rightarrow{ }^{4} \mathrm{~A}_{2} \mathrm{~g}$ & \\
\hline & 879 & 11,377 & 4 & ${ }^{4} \mathrm{~T}_{1} \mathrm{~g} \rightarrow{ }^{4} \mathrm{~T}_{2} \mathrm{~g}$ & \\
\hline \multirow{6}{*}[\mathrm{Ni}(\mathrm{HL})_{2}(\mathrm{N}_{3})_{2}]{$\cdot 3 \mathrm{H}_{2} \mathrm{O}$} & 281 & 35,587 & 2218 & Intra-ligand & \multirow{6}{*}{ Oh } \\
\hline & 347 & 28,818 & 1152 & Intra-ligand & \\
\hline & 365 & 27,397 & 842 & Intra-ligand & \\
\hline & 426 & 23,474 & 323 & ${ }^{3} \mathrm{~A}_{1} \mathrm{~g} \rightarrow{ }^{3} \mathrm{~T}_{2} \mathrm{~g}(\mathrm{P})$ & \\
\hline & 792 & 15,942 & 10 & ${ }^{3} \mathrm{~A}_{1} \mathrm{~g} \rightarrow{ }^{3} \mathrm{~T}_{1} \mathrm{~g}$ & \\
\hline & 879 & 11,377 & 2 & ${ }^{3} \mathrm{~A}_{1} \mathrm{~g} \rightarrow{ }^{3} \mathrm{~T}_{2} \mathrm{~g}$ & \\
\hline \multirow{3}{*}[\mathrm{Zn}(\mathrm{HL})_{2}(\mathrm{N}_{3})_{2}]{$\cdot 3 \mathrm{H}_{2} \mathrm{O}$} & 286 & 34,965 & 2278 & Intra-ligand & \multirow{3}{*}{$\mathrm{Oh}$} \\
\hline & 345 & 28,986 & 1489 & Intra-ligand & \\
\hline & 354 & 28,249 & 962 & Intra-ligand & \\
\hline \multirow{3}{*}[\mathrm{Cd}(\mathrm{HL})_{2}(\mathrm{N}_{3})_{2}]{$\cdot 2 \mathrm{H}_{2} \mathrm{O}$} & 286 & 34,965 & 2276 & Intra-ligand & \multirow{3}{*}{$\mathrm{Oh}$} \\
\hline & 345 & 28,986 & 1485 & Intra-ligand & \\
\hline & 354 & 28,249 & 960 & Intra-ligand & \\
\hline \multirow{3}{*}[\mathrm{Hg}(\mathrm{HL})_{2}(\mathrm{N}_{3})_{2}]{$\cdot 3 \mathrm{H}_{2} \mathrm{O}$} & 284 & 35,211 & 2274 & Intra-ligand & \multirow{3}{*}{ Oh } \\
\hline & 345 & 28,986 & 2085 & Intra-ligand & \\
\hline & 354 & 28,249 & 1266 & Intra-ligand & \\
\hline
\end{tabular}


transitions and suggested geometries. The electronic spectra for all mixed ligand complexes displayed three absorption peaks in the ultraviolet region. The first peak at range $(281-297) \mathrm{nm}(35587-33670) \mathrm{cm}^{-1}\left(\varepsilon_{\max }=\right.$ $\left.2370-\varepsilon_{\max }=2218\right) \mathrm{mol}^{-1} \cdot \mathrm{L} \cdot \mathrm{cm}^{-1}$, the second peak at $(345,347) \mathrm{nm}(28986,28818) \mathrm{cm}^{-1}\left(\varepsilon_{\max }=1152-\varepsilon_{\max }=\right.$ 2278) $\mathrm{mol}^{-1} \cdot \mathrm{L}^{-\mathrm{cm}^{-1}}$, the third peak at $(354-365) \mathrm{nm}(28249-27397) \mathrm{cm}^{-1}\left(\varepsilon_{\max }=842-\varepsilon_{\max }=1324\right)$ $\mathrm{mol}^{-1} \cdot \mathrm{L} \cdot \mathrm{cm}^{-1}$ were attributed to intra-ligand $\pi \rightarrow \pi^{*}, \mathrm{n} \rightarrow \pi^{*}$, and $\mathrm{n} \rightarrow \pi^{*}$, respectively [25] which exhibited bath chromic shift or hypsochromic shift when it comparison with that of free ligand (HL).

\subsection{5. $\left[\mathrm{Mn}(\mathrm{HL})_{2}\left(\mathrm{~N}_{3}\right)_{2}\left(\mathrm{H}_{2} \mathrm{O}\right)_{2}\right] \cdot \mathrm{H}_{2} \mathrm{O}$}

The UV-Vis spectrum of Mn(II) complex, displayed three additional absorption peaks. The first peak at (475) $\mathrm{nm}(21053) \mathrm{cm}^{-1}\left(\varepsilon_{\max }=208\right) \mathrm{mol}^{-1} \cdot \mathrm{L} \cdot \mathrm{cm}^{-1}$, the second peak at $(638) \mathrm{nm}(15674) \mathrm{cm}^{-1}\left(\varepsilon_{\max }=41\right) \mathrm{mol}^{-1} \cdot \mathrm{L}^{-} \mathrm{cm}^{-1}$, the third peak at (794) nm (12594) $\mathrm{cm}^{-1}\left(\varepsilon_{\max }=7\right) \mathrm{mol}^{-1} \cdot \mathrm{L}^{6} \mathrm{~cm}^{-1}$ were attributed to (d-d) spin-forbidden electronic transition type ${ }^{6} \mathrm{~A}_{1} \mathrm{~g} \rightarrow{ }^{4} \mathrm{~T}_{2} \mathrm{~g}_{(\mathrm{G})},{ }^{6} \mathrm{~A}_{1} \mathrm{~g} \rightarrow{ }^{4} \mathrm{~T}_{1} \mathrm{~g}_{(\mathrm{G})}$, and ${ }^{6} \mathrm{~A}_{1} \mathrm{~g} \rightarrow{ }^{4} \mathrm{Eg}(\mathrm{D})$, confirming octahedral geometry about Mn(II) [27].

\subsection{6. $\left[\mathrm{Co}(\mathrm{HL})_{2}\left(\mathrm{~N}_{3}\right)_{2}\right] \cdot 2 \mathrm{H}_{2} \mathrm{O}$}

The electronic spectrum of Co(II) complex, showed two additional absorption peaks. The first peak at (650) nm $(15,387) \mathrm{cm}^{-1}\left(\varepsilon_{\max }=13\right) \mathrm{mol}^{-1} \cdot \mathrm{L} \cdot \mathrm{cm}^{-1}$, and the second peak at (879) $\mathrm{nm}(11377) \mathrm{cm}^{-1}\left(\varepsilon_{\max }=4\right) \mathrm{mol}^{-1} \cdot \mathrm{L} \cdot \mathrm{cm}^{-1}$ were attributed to (d-d) spin-allowed electronic transition type ${ }^{4} \mathrm{~T}_{1} \mathrm{~g} \rightarrow{ }^{4} \mathrm{~A}_{2} \mathrm{~g}$, and ${ }^{4} \mathrm{~T}_{1} \mathrm{~g} \rightarrow{ }^{4} \mathrm{~T}_{2} \mathrm{~g}$, respectively, characteristic octahedral geometry around Co(II) [28].

\subsection{7. $\left[\mathrm{Ni}(\mathrm{HL})_{2}\left(\mathrm{~N}_{3}\right)_{2}\right] \cdot \mathrm{H}_{2} \mathrm{O}$}

The UV-Vis spectrum of Ni(II) complex, displayed three additional absorption peaks. The first peak at (426) nm $(23,474) \mathrm{cm}^{-1}\left(\varepsilon_{\max }=323\right) \mathrm{mol}^{-1} \cdot \mathrm{L}^{2} \mathrm{~cm}^{-1}$, the second peak at $(792) \mathrm{nm}(15942) \mathrm{cm}^{-1}\left(\varepsilon_{\max }=10\right) \mathrm{mol}^{-1} \cdot \mathrm{L}^{\mathrm{c}} \mathrm{cm}^{-1}$, the third peak at $(879) \mathrm{nm}(11,377) \mathrm{cm}^{-1}\left(\varepsilon_{\max }=2\right) \mathrm{mol}^{-1} \cdot \mathrm{L}^{-1} \mathrm{~cm}^{-1}$ were due to (d-d) spin-allowed electronic transition type ${ }^{3} \mathrm{~A}_{1} \mathrm{~g} \rightarrow{ }^{3} \mathrm{~T}_{1} \mathrm{~g}(\mathrm{P}),{ }^{3} \mathrm{~A}_{1} \mathrm{~g} \rightarrow{ }^{3} \mathrm{~T}_{1} \mathrm{~g}$, and ${ }^{3} \mathrm{~A}_{1} \mathrm{~g} \rightarrow{ }^{3} \mathrm{~T}_{2} \mathrm{~g}$, respectively, which were a good agreement for octahedral geometry of $\mathrm{Ni}(\mathrm{II})$ complexes [29].

\subsection{8. $\left[\mathrm{Zn}(\mathrm{HL})_{2}\left(\mathrm{~N}_{3}\right)_{2}\right] \cdot 3 \mathrm{H}_{2} \mathrm{O},\left[\mathrm{Cd}(\mathrm{HL})_{2}\left(\mathrm{~N}_{3}\right)_{2}\right] \cdot 2 \mathrm{H}_{2} \mathrm{O}$, and $\left.\mathrm{Hg}(\mathrm{HL})_{2}\left(\mathrm{~N}_{3}\right)_{2}\right] \cdot 3 \mathrm{H}_{2} \mathrm{O}$}

The electronic spectra of $\mathrm{Zn}(\mathrm{II}), \mathrm{Cd}(\mathrm{II})$, and $\mathrm{Hg}(\mathrm{II})$, respectively show no absorption peaks in the visible region, indicating ( $\mathrm{d}^{10}$-system) for $\mathrm{Zn}(\mathrm{II})$, Cd(II), and $\mathrm{Hg}(\mathrm{II})$, that is mean no d-d electronic transition happened [27].

\subsection{Biological Activity of the Ligand (HL) and Mixed Ligand Complexes}

Indicating that the new ligand and its mixed ligand complexes exhibited antibacterial activity against four kinds of bacterial: Staphylococcus aureus, Bacillus, Escherichia coli, and Pseudomonad aureus respectively, in Table 6, Figure 1 and Figure 2. The enhanced activity of the complexes can be explained on the bases of Overtone's concept and Tweedy's chelation theory [30] [31].

Table 6. Showed the inhibition circle diameter in millimeter for the bacteria after 24 hour in cubation paid (37 $\left.{ }^{\circ} \mathrm{C}\right)$ for ligand (HL) and its mono and mated complexes.

\begin{tabular}{ccccc}
\hline Compounds & E. coli & Pesudomonas & Bacillus & Staphylococcus \\
\hline DMSO & 5 & 7 & - & 5 \\
Ligand $(\mathrm{HL})$ & 13 & 14 & 12 & 11 \\
{$\left[\mathrm{Mn}(\mathrm{HL})_{2}\left(\mathrm{~N}_{3}\right)_{2}\left(\mathrm{H}_{2} \mathrm{O}\right)_{2}\right] \cdot \mathrm{H}_{2} \mathrm{O}$} & - & 11 & - & 15 \\
{$\left[\mathrm{Co}(\mathrm{HL})_{2}\left(\mathrm{~N}_{3}\right)_{2}\right] \cdot 2 \mathrm{H}_{2} \mathrm{O}$} & 14 & - & - & 11 \\
{$\left[\mathrm{Ni}(\mathrm{HL})_{2}\left(\mathrm{~N}_{3}\right)_{2}\right] \cdot 3 \mathrm{H}_{2} \mathrm{O}$} & 13 & 10 & 21 & 11 \\
{$\left[\mathrm{Zn}(\mathrm{HL})_{2}\left(\mathrm{~N}_{3}\right)_{2}\right] \cdot 3 \mathrm{H}_{2} \mathrm{O}$} & 13 & 11 & 32 & 18 \\
{$\left[\mathrm{Cd}(\mathrm{HL})_{2}\left(\mathrm{~N}_{3}\right)_{2}\right] \cdot 2 \mathrm{H}_{2} \mathrm{O}$} & 16 & 16 & 26 & 12 \\
{$\left[\mathrm{Hg}(\mathrm{HL})_{2}\left(\mathrm{~N}_{3}\right)_{2}\right] \cdot 3 \mathrm{H}_{2} \mathrm{O}$} & 26 & 11 & & 11 \\
\hline
\end{tabular}



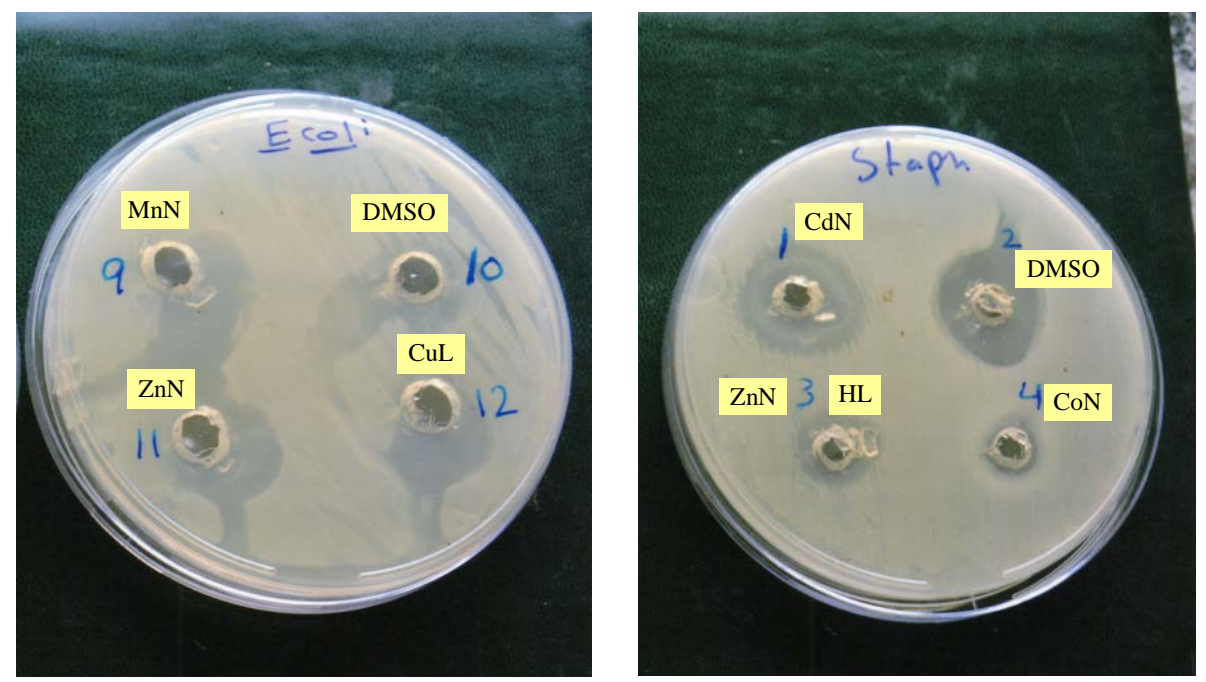

Figure 1. Inhibition diameter for ligands and complexes E. coli = Escherichia coli, Staph = Staphylococcus aureus.
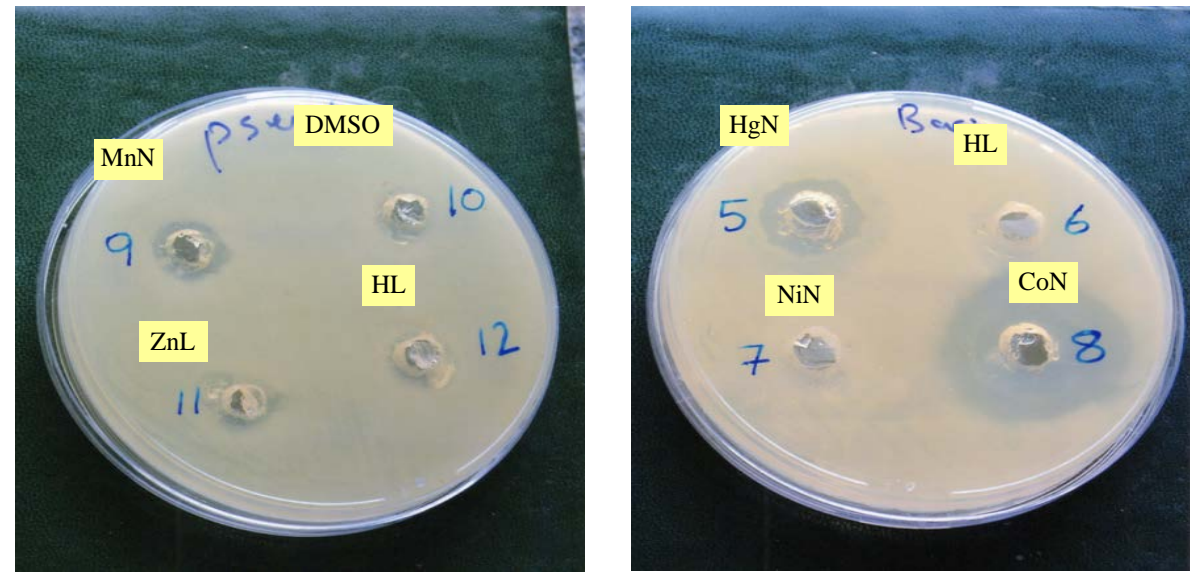

Figure 2. Inhibition diameter for ligands and complexes Pse = Pesudomonas, Bacil = Bacillus.

\section{Conclusions and the Proposed Molecular Structure for All Prepared Complexes}

According to the characterization data for new Schiff base (HL) derived from 4-aminoantipyrine with glyoxylic acid, and its mixed ligand complexes based Schiff base liagnd HL primary ligand and azide ion $\left(\mathrm{N}_{3}^{-}\right)$ (co-ligand), by FT-IR, UV-Vis, atomic absorption, ${ }^{1} \mathrm{H}$ NMR, ${ }^{13} \mathrm{C}$ NMR, magnetic susceptibility, molar conductivity, elemental microanalysis, chloride content alonge with melting point, we found that:

1) The Schiff base (HL) in mixed ligand complexes behaved bidentatc ligand through its azomethine nitrogen, oxygen atom of $\mathrm{C}=\mathrm{O}$ group of five member ring with the central metal ions: $\mathrm{Co}(\mathrm{II}), \mathrm{Ni}(\mathrm{II}), \mathrm{Zn}(\mathrm{II}), \mathrm{Cd}(\mathrm{II})$ and $\mathrm{Hg}(\mathrm{II})$ forming complexes with molecular formula: $\left[\mathrm{M}(\mathrm{HL})_{2}\left(\mathrm{~N}_{3}\right)_{2}\right] \cdot \mathrm{mH}_{2} \mathrm{O}$ where: $\left(\mathrm{M}^{\mathrm{II}}=\mathrm{Co}, \mathrm{Cd} ; \mathrm{m}=2\right),\left(\mathrm{M}^{\mathrm{II}}=\right.$ $\mathrm{Ni}, \mathrm{Zn}, \mathrm{Hg} ; \mathrm{m}=3$ ), except in Mn(II) complex, Schiff base (HL) behaved monodentatc ligand via azomethine nitrogen forming complex with molecular formula $\left[\mathrm{Mn}(\mathrm{HL})_{2}\left(\mathrm{~N}_{3}\right)_{2}\right] \cdot \mathrm{H}_{2} \mathrm{O}$.

2) The octahedral geometrical structure was suggested for all prepared complexes based on the characterization data for all technique.

3) The antibacterial study showed that the complexes were more toxic to the strain of bacteria taken under study than the Schiff base ligand (HL).

\section{References}

[1] Mehta, B.H. and Chavan, V.L. (2011) X-Ray, Thermal and Biological Studies of Ru(III), Rh(III) and Pd(II) Schiff 
Base Metal Complexes. Research Journal of Chemistry and Environment, 15, 57-61.

[2] Raman, N., Raja, S.J. and Salkthivel, A. (2009) Transition Metal Complexes with Schiff-Base Ligands: 4-Aminoantipyrine Based Derivatives-A Review. Journal of Coordination Chemistry, 62, 691-709. http://dx.doi.org/10.1080/00958970802326179

[3] Rosu, T. Pahontu, E., Maxim, C., Georgescu, R., Stanica, N. and Gulea, A. (2011) Some New Cu(II) Complexes Containing an on Donor Schiff Base: Synthesis, Characterization and Antibacterial Activity. Polyhedron, 30, 154-162. http://dx.doi.org/10.1016/j.poly.2010.10.001

[4] Mohamed, G.G., Omar, M.M. and Ibrahim, A.A. (2009) Biological Activity Studies on Metal Complexes of Novel Tridentate Schiff Base Ligand. Spectroscopic and Thermal Characterization. European Journal of Medicinal Chemistry, 44, 4801-4812. http://dx.doi.org/10.1016/j.ejmech.2009.07.028

[5] Elemike, E.E. Oviawe A.P. and Otuokere, I.E. (2011) Potentiation of the Antimicrobial Activity of 4-Benzylimino-2,3Dimetyl-1-phenylpyrazal-5-one by Metal Chelation. Research Journal of Chemical Sciences, 1, 6-11.

[6] Agarwal, R.K., Singh, I. and Sharma, D.K. (2006) Synthesis, Spectral, and Biological Properties of Copper(II) Complexes of Thiosemicarbazones of Schiff Bases Derived from 4-Aminoantipyrine and Aromatic Aldehydes. Bioinorganic Chemistry and Applications, 2006, 1-10.

[7] Acheson, R.M. (2009) Introduction to Heterocyclic Compounds. 4th Edition, Wiley, New York.

[8] Gopalakrishman, S. and Joseph, J. (2009) Antifungal Activities of Copper (II) with Biosensitive Macrocyclic Shiff Base Ligands Derived from 4-Aminoantipyrien Derivatives. Microbiology, 37, 141-146.

[9] Mishchenco, A.V. Lukov V.V. and Popov, L.D. (2011) Synthesis and Physico-Chemical Study of Complexation of Glyoxylic Acid Aroylhydrazones with Cu(II) in Solution and Solid Phase. Journal of Coordination Chemistry, 64, 1963-1976. http://dx.doi.org/10.1080/00958972.2011.585640

[10] Kramer, D.N., Klein, N. and Baselice, R.A. (1999) Quantitative Determination of Glyoxylic Acid. Analytical Chemistry, 31, 250-252. http://dx.doi.org/10.1021/ac60146a028

[11] Sokol, H.A. (1977) Determination of Pyruvic and Glyoxylic Acids in the Presence of Acetaldehyde. Analytica Chimica Acta, 89, 407-408. http://dx.doi.org/10.1016/S0003-2670(01)84741-3

[12] Tharmaraj, P., Kodimunthiri, D., Sheela, C.D. and Shanmugapriya, C.S. (2009) Synthesis, Spectral Studies and Antibacterial Activity of $\mathrm{Cu}(\mathrm{II}), \mathrm{Co}(\mathrm{II})$ and $\mathrm{Ni}(\mathrm{II})$ Complexes of 1-(2-Hydroxyphenyl)-3-phenyl-2-propen-1-one) $\mathrm{N}^{2}$-[(3,5dimethyl-1H-pyrazol-1-yl)methyl] Hydrazone. Journal of the Serbian Chemical Society, 74, 927-938. http://dx.doi.org/10.2298/JSC0909927T

[13] Suresh, M.S. and Prakash, V. (2010) Preparation and Characterization of Cr(III), Mn(II), Co(II), Ni(II), Cu(II), Zn(II) and Cd(II) Chelates of Schiffs Base Derived from 4-Aminoantipyrine. International Journal of Physical Sciences, 5, 2203-2211.

[14] Anupama, B., Padmaja, M. and Kumari, C.G. (2012) Synthesis, Characterization, Biological Activity and DNA Binding Studies of Metal Complexes with 4-Aminoantipyrine Schiff Base Ligand. E-Journal of Chemistry, 9, 389-400. http://dx.doi.org/10.1155/2012/291850

[15] Raman, N., Sobha, S. and Selvaganapathy, M. (2012) Probing the DNA Binding Mode of Transition Metal Based Biologically Active Compounds: Validation by Spectroscopic Methods. International Journal of Pharma and Bio Sciences, 3, 251-268.

[16] Manjula, B. and Antony, S.A. (2013) Arsenic Induced Biochemical Changes in Perna viridis as Potential Biomarkers in Metal Pollution. Asian Journal of Biochemical and Pharmaceutical Research, 3, 168-178.

[17] Wang, X.Y., Wang, Z.M. and Gao, S. (2008) Constructing Magnetic Molecular Solids by Employing Three-Atom Ligands as Bridges. Chemical Communications, 2008, 281-294. http://dx.doi.org/10.1039/B708122G

[18] Ju, Z.F., Yao, Q.X., Wu, W. and Zhang, I. (2008) Strong Electron-Accepting Methyl Viologen Dication Confined in Magnetic Hosts: Synthesis, Structural, Characterization, Change-Transfer and Magnetic Properties, of $\left[(\mathrm{MV})_{2}[\mathrm{Ni}-\right.$ $\left.\left.(\mathrm{SCN})_{5}\right] \cdot \mathrm{Cl} \cdot 2 \mathrm{H}_{2} \mathrm{O}\right]_{\mathrm{n}}$ and $\left[(\mathrm{MV})\left[\mathrm{M}\left(\mathrm{N}_{3}\right)_{2}(\mathrm{SCN})_{2}\right]\right](\mathrm{M}=\mathrm{Mn}, \mathrm{Co})$. Dalton Transactions, 355-362. http://dx.doi.org/10.1039/B710628A

[19] Belaid, S., Landreau, A., Djebbar, S., Benali-Baitich, O., Bouet, G. and Bouchara, J. (2008) Synthesis, Characterization and Antifungal Activity of a Series of Manganese(II) and Cupper(II) Complexes with Ligands Derived from Reduced N,N-O-phenylene bis(salicylideneimine). Journal of Inorganic Biochemistry, 102, 63-69. http://dx.doi.org/10.1016/j.jinorgbio.2007.07.001

[20] El-Asmy, A.A., Al-Ansi, T.Y. and Shaiba, Y.M. (1989) Chelated Complexes of Cadmium(II), Cobalt(II), Copper(II), Mercury(II), Nickel(II), Uranyl(II) and Zinc(II) with Benzil bis(4-phenylthiosemicarbazone). Transition Metal Chemistry, 14, 446-452. http://dx.doi.org/10.1007/BF01092587

[21] Socrates, G. (1980) Infrared Characteristic Group Frequencies. Wiley, New York. 
[22] Raj, K.D. and Sharad, K.M. (2011) Synthesis, Spectroscopic (IR, Electronic, Fab-Mass, and PXRD), Magnetic and Antimicrobial Studies of New Iron(III) Complexes Containing Shiff Bases and Substituted Benzoxazole Ligands. Journal of Coordination Chemistry, 64, 2292-2301. http://dx.doi.org/10.1080/00958972.2011.594886

[23] Nakamoto, K. (1978) Infrared and Raman Spectra of Inorganic and Coordination Compounds. 3rd Edition, Wiley, New York.

[24] Cowely, A.R. Dilworth, J.R. Donnelly, P.S. and Whilte, J.M. (2006) Copper Complexes of Thiosemicarbazone-Pyridylhydrazine (THYNIC) Hybrid Ligands: A New Versatile Potential Bifunctional Chelator for Copper Radiopharmaceuticals. Inorganic Chemistry, 45, 496-498. http://dx.doi.org/10.1021/ic0514492

[25] Al-Hamdani, A.A.S. and Shaker, S.A. (2011) Synthesis, Characterization, Structural Studies and Biological Activity of a New Schiff Base-Azo Ligand and Its Complexation with Selected Metal Ions. Oriental Journal of Chemistry, 27, 835-845.

[26] Lever, A.B.P. (1984) Inorganic Electronic Spectroscopy. 2nd Edition, Elsevier, New York.

[27] Huheey, J.E. (1994) Inorganic Chemistry: Principles of Structure and Reactivity. Harper and Row Publisher, New York.

[28] Awetz, J., Melnick, P. and Delbrgs, A. (2007) Medical Microbiology. 4th Edition, McGraw Hill, New York.

[29] Priya, N.P., Arunachalam, S.V., Sathya, N., Chinnusamy, V. and Jayabalakrishnan, C. (2009) Catalytic and Antimicrobial Studies of Binuclear Ruthenium(III) Complexes, Containing Bis- $\beta$-Diketones. Transition Metal Chemistry, 34, 437-445. http://dx.doi.org/10.1007/s11243-009-9214-z

[30] Jayablakrishnan, C. and Natarajan, K. (2002) Synthesis, Characterization, and Biological Activities of Ruthenium(II) Carbonyl Complexes Containing Bifunctional Tridentate Schiff Bases. Transition Metal Chemistry, 27, 75-79. http://dx.doi.org/10.1023/A:1013437203247

[31] Tweedy, B.G. (1964) Plant Extracts with Metal Ions as Potential Antimicrobial Agents. Phytopatology, 55, 910-918. 